\title{
Simulation Analysis of Knowledge Transfer Behaviour Based on Small World Network Model
}

\author{
Yang $\mathrm{Bo}^{1}$ and $\mathrm{Yu} \mathrm{She}^{2}$ \\ 1Jiangxi University of Finance \& Economics, School of Information Management, \\ Nanchang, China \\ 2 Jiangxi Normal University, Nanchang, China \\ yangbo@jxufe.edu.cn; 741253400@qq.com
}

\begin{abstract}
This paper constructs the knowledge transfer simulation mode based on small world network model. It uses the average path length and clustering coefficient to stand for AC frequency and aggregation degree among knowledge network nodes and studies the node's ability to release and absorb as well as the knowledge transfer effect by trust degree. Simulation results show that improving the AC frequency and aggregation degree of nodes, enhancing node's ability to transfer knowledge can ensure transfer frequency in organization reaching a high level.
\end{abstract}

Keywords: Small World Network; Knowledge Network; Knowledge Transfer; MultiAgent Mode

\section{Introduction}

Social networks are the society that has an independent ability to judge the subject as a node, the interaction between the body and the formation of networks as side. The social network analysis is based on inter subjective interrelated specific features, analysis and interpretation of the actors of the same overall system behavior. Knowledge network is in the social network of domain knowledge and knowledge activities in connection with the construction of the network [1], a large number of statistical data show that, the social network model has the small world network model [2-5]. Small world network is a highly aggregative but longer average path regular networks and having a low intensity but a network between random network with short average path, it also has a degree and short path feature set high, it has special effect in the network structure and network communication function [6].

In the era of knowledge economy, knowledge creation, knowledge dissemination and diffusion, knowledge utilization are all important factors of economic growth, knowledge network in real life are embedded in social networks, facing the fast-changing market, knowledge network dynamic alliance and other forms of known as the rational choice of enterprises with the environment[7]. Division of labor and knowledge sharing in a dynamic social network organization has become an important channel for the characteristics of Inter Organizational Knowledge Transfer, the knowledge network construction has become an important channel for the dissemination of knowledge, knowledge transfer and innovation. In the network environment of the knowledge transfer process, the complex interactions between sender and recipient of knowledge is affected by the network structure and mode. Research at present in the network main body of knowledge transfer are built on the comparative static analysis, such as network subject to the microscopic interaction, or pay attention to network among the main macro behavior patterns. Due to the Embedded feature of knowledge resources acquisition in network structure [8-11], so it is difficult to distinguish what kind of network structure play a role 
in the network organization, which kind of network structure more adapt to network node behavior ability. Network node level of knowledge, knowledge of the release and absorption ability and trust relationship of nodes affect the entire network organization knowledge level and knowledge transfer efficiency. In the knowledge transfer of network analysis process, each node in the network can be seen as having agent learning, judgment, decision making, representing different body of knowledge in social networks, together constitute the complex adaptive multi-agent system(CAS) based on agent system theory. Links between nodes in knowledge transfer network can enhance the overall level of knowledge, therefore, clustering coefficient and average path network can be used as an important index to measure the network knowledge transfer and cooperation degree.

Based on the complex network theory, the paper adopts multi-agent modeling and simulation of complex adaptive system and establishes the small world network model in multi-agent simulation platform, NetLogo, then constructs simulation model of knowledge transfer behavior in small world network.

\section{The Basic Idea of Knowledge Transfer Behavior Model in Small World Network}

\subsection{The Question Analysis of Knowledge Transfer Behavior Model in Small World Network}

The purpose of this paper is to study knowledge transfer behavior regulation of small world network, since that can't be tested in real world. Consequently, adopting computer construction model and simulation method is an effective way. The basic idea is that as a result of the complexity of social network system and the complex features of knowledge transfer and evolution among subjects, multi-agent-based modeling and simulation method to study the knowledge transfer behavior can be the best way to apply for the study of complex system. Knowledge transfer network is a complex network, which has the feature of small world network [17]. Thus, complex network theory can be used to construct small world network model. The foundation of knowledge transfer is the interaction of knowledge among network subjects, and knowledge dissemination and interaction process is a typical system dynamic process, $[16,18]$ so knowledge transfer dynamic can be applied to set the rule of knowledge transfer. The following Figure 1 shows about it.

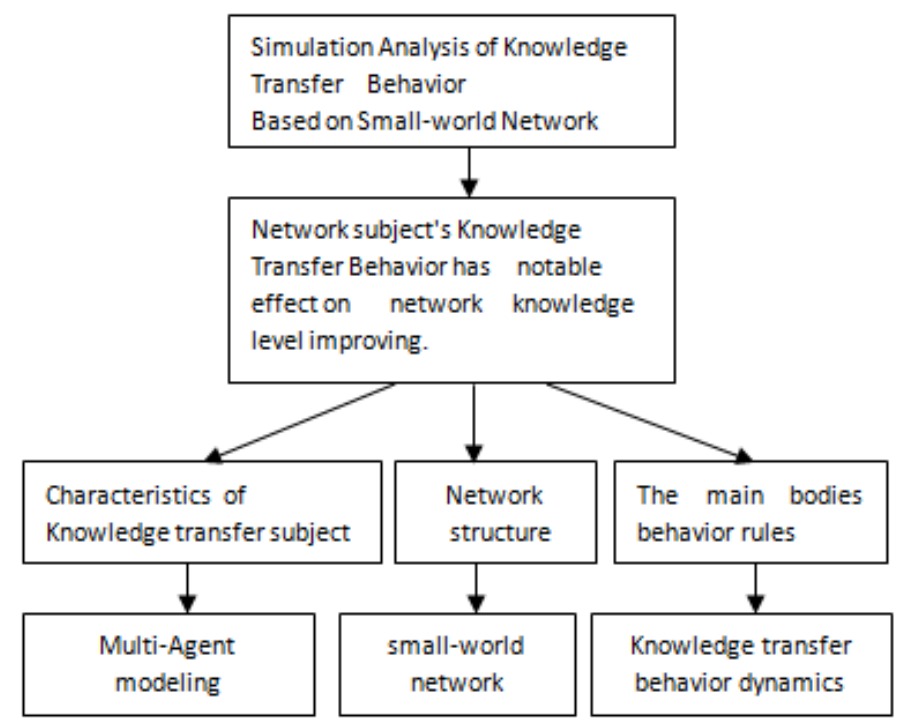

Figure 1. Analysis about Construction Modeling of Knowledge Transfer Behavior in Small Network 
Agent-Based Modeling and Simulation [20]( (ABMS) is a construction modeling way from bottom to top, a macroscopic behavior to describe complex system by depicting the behavior of agent unit and its mutual each other(including environment). Based on different units in system, the method identifies the object of construction modeling and extracts key attributes to construct unit agent modeling, setting attributes, behaviors, interaction rules and relative constraint conditions of agent to study the effect of unit behavior to the whole system by operating the system. The method applied ABMS to construct simulation system is called Multi-Agent System. ABMS method can simulate unit heterogeneity well, but specification and complexity of the modeling are constrained by obtained resource and statistics, meanwhile simulation operation would cost high calculating resource and modeling verification is very difficult.

Complex network theory originated from Stanley Milgram, a sociologist from Harford University, in 1967, who designed a chain letter experiment and offered Six Degrees of Separation, that is Small World Phenomenon. The phenomenon reflexes a common object regulation, which means that due to the coming of era of network information, the relationship among people has become socialized. Everyone can have a certain relationship with others by Six Degrees of Separation (Milgram S, 1967). In order to explain the small world effect by network picture, Watts and Strogatz published a first paper about constructing mathematic modeling of small world network based on the study to regular network and random network theory and offered famous conception WS small world network (SWN) (Watts D. J. \& Strogatz S.H, 1998). In the modeling of WS SWN, small world network has both high degree of aggregation and short path, thus, it has special effect in network structure and network spreading.

Knowledge transfer behavior dynamics [18] focuses on describing knowledge interaction process among units, which involves the whole process from releasing, acquiring, absorbing knowledge to new knowledge completing. This modeling usually assumes that the main participators are consisted of subjects having certain social network relationship, and the knowledge holding by the subjects of knowledge transfer is expressed by continuous or discrete numerical value. The subjects can begin knowledge transfer process under certain initial conditions: in a knowledge interaction, subjects transfer knowledge based on certain rules and trusted relation, while knowledge sender and receiver would transfer, receive and create knowledge according to their own knowledge releasing ability respectively. After repeating several times of transfer and reflection, the whole knowledge level among subjects would improve. In brief, the main research object of knowledge transfer behavior dynamics is knowledge interaction rules of subjects. This paper assumes that behavior dynamics modeling of subjects is isomorphic and completely mixed and simplifies special attributes among subjects.

\subsection{Modeling Framework of Knowledge Transfer Behavior based on Multi-agent Small World Network}

This paper offers modeling and simulation method of knowledge transfer behavior based on multi-agent small world network, an integrated way suited to the modeling of knowledge transfer behavior in small world network, by the use of comparing the method of agent-based modeling and simulation, complex network and knowledge transfer dynamics. The basic idea of this method is simulating the subject of knowledge transfer network to the agent of computer, simulating knowledge transfer behavior among subjects to the attribute and behavior of agent and introducing modeling of knowledge transfer dynamics to describe, depicting the relationship among subjects through modeling of small world network in computer. The method can solve effectively about how to use microcosmic knowledge transfer behavior of subjects to change knowledge level of macrocosmic knowledge network.

Setting certain modeling parameter based of real social environment, like construction of small world network, rules of knowledge interaction, initial state of system, knowledge 
releasing, absorbing ability and trusting relationship and so on, and using modeling and simulation method of knowledge transfer behavior based on multi-agent small world network can study all kinds of influencing factors to the behavior and simulation process of knowledge transfer. Guiding by all of above, the general framework of knowledge transfer behavior based on multi-agent small world network can be showed in Figure 2 .

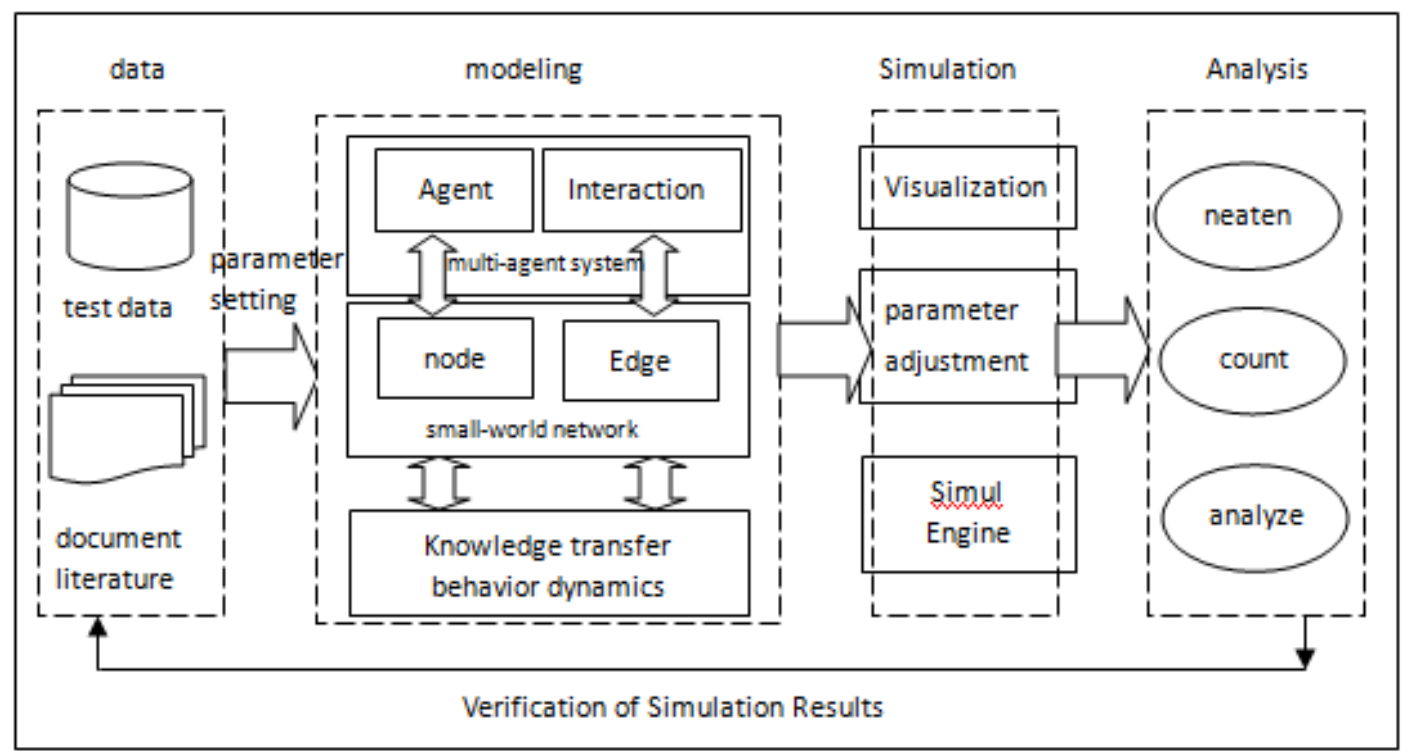

Figure 2. Knowledge Transfer Behavior Model Framework Based on the Agent Small World Network

\section{Construction of Modeling based on NetLogo Small World Network}

NetLogo [19] is a programming construction environment used to simulate natural and social phenomenon. In 1999, Uri Wilensky first put forward this instrument and then developed it in Connected Learning and Computer-Based Modeling center in Northwestern University. Its software, modeling and relative document can be downloaded freely from its official website. NetLogo especially suits for modeling of complex system, which simulates as the change of time. It has many types of agent modeling, like turtle, patch, and link, which can give out commands to hundreds of agent. It is possible to research microscopically the relation between unit behavior and macroscopic mode, which comes from the interaction among units. The bottom of NetLogo is realized by Java program language and can be operated on all main platforms, including Mac, Windows and Linux as well as on browser as Java Applets. NetLogo has detailed document and teaching material. It also has a model base, which includes many written simulation models. These model covers many fields of natural and social science, including biology and medicine, physics and chemistry, math and computer science, economics and social psychology etc.

Knowledge network, which constructs on social network and has the feature of dynamic division of labor and knowledge sharing, has become an important channel to spread, transfer and create knowledge among organizations. By communicating on nodes of network, knowledge finally form knowledge sharing and knowledge transfer and offer driving force to knowledge innovation on network nodes. The relation among every nodes of knowledge transfer network can improve the whole knowledge level of organization, therefore the distribution, clustering coefficient and average path in network can be very important indicators to measure knowledge transfer of network and cooperation degree [12].

Specific analysis is as follows: 
- The degree of the node refers to the node associated with the number of edges that connect the node to other nodes. Degree distribution refers to the degree distribution of nodes in the network have, namely node selected In the random consistent principle of the degree of K probability, generally denoted as $\mathrm{P}(\mathrm{k})$.

- The clustering coefficient represents the clustering characteristic of network, namely the cluster characteristics. Assuming network nodes i and $k_{i}$ edges relative to practice, that is connected with the other $k_{i}$ nodes. $k_{i}$ nodes in between there may be up $\frac{k(k-1)}{2}$ edges, If these $k_{i}$ nodes exist between the actual number of edges $E_{i}$. Then $E_{i}$ and the total possible number of edges $\frac{k(k-1)}{2}$ ratio became the clustering coefficient $C_{i}$ of the node i, namely $C_{i}=\frac{2 E_{i}}{k_{i}\left(k_{i}-1\right)}$. All nodes in the network to take the average of the clustering coefficient can be obtained by the whole network clustering coefficient $\mathrm{C}$, namely $C=\frac{1}{N} \sum_{i=1}^{N} C_{i}$,where $\mathrm{N}$ is the total number of nodes in the network.

- Average shortest path of the network can be used to describe the connectivity of the network better. Two nodes in the network, the distance between $\mathrm{i}$ and $\mathrm{j} d_{i j}$ for connecting these two nodes number of edges on the shortest path. The average shortest path length of network is defined as the average value between any 2 nodes of the average shortest path, namely

$$
L=\frac{1}{\frac{1}{2} N(N-1)} \sum_{i>j} d_{i, j}
$$

where $\mathrm{N}$ is the total number

of nodes in the network.

Characteristics of the traditional nearest neighbor coupled regular networks with high clustering, but does not have the characteristics of small world. While the ER random network with small world characteristics, but no high clustering characteristics. Therefore, these two traditional network models are not well represent the actual real network. WS small-world network model to be between these two networks, both small-world and clustering characteristics, can be a good representation of the real world. Small-world network model is constructed from a regular network algorithm to random networks by rewiring probability $\mathrm{p}$ changes from zero to one, construct network model with small world effect. The small world network model of the random rewiring structure as shown in Figure 3.

- Create a containing $\mathrm{n}$ points of the nearest neighbor coupled regular networks, around a ring network, in which each node is associated with it a link, a total of $\mathrm{K}$ adjacent points, namely about each side of $\mathrm{k}=0.5^{*} \mathrm{~K}$ nodes, $\mathrm{k}$ is a natural number, see Figure 3(a).

- Random with probability $\mathrm{p}$ of each reconnect to the network side, the side of one end of the remained unchanged, while the other end of the network is connected to another node randomly selected in the connection rules to exclude their connections and duplicate connection, see Figure 3(b).

- In the small-world network model, $\mathrm{p}=0$ corresponds to the rule network, $\mathrm{p}=1$ corresponds to the completely random network, see Figure 3(c). 

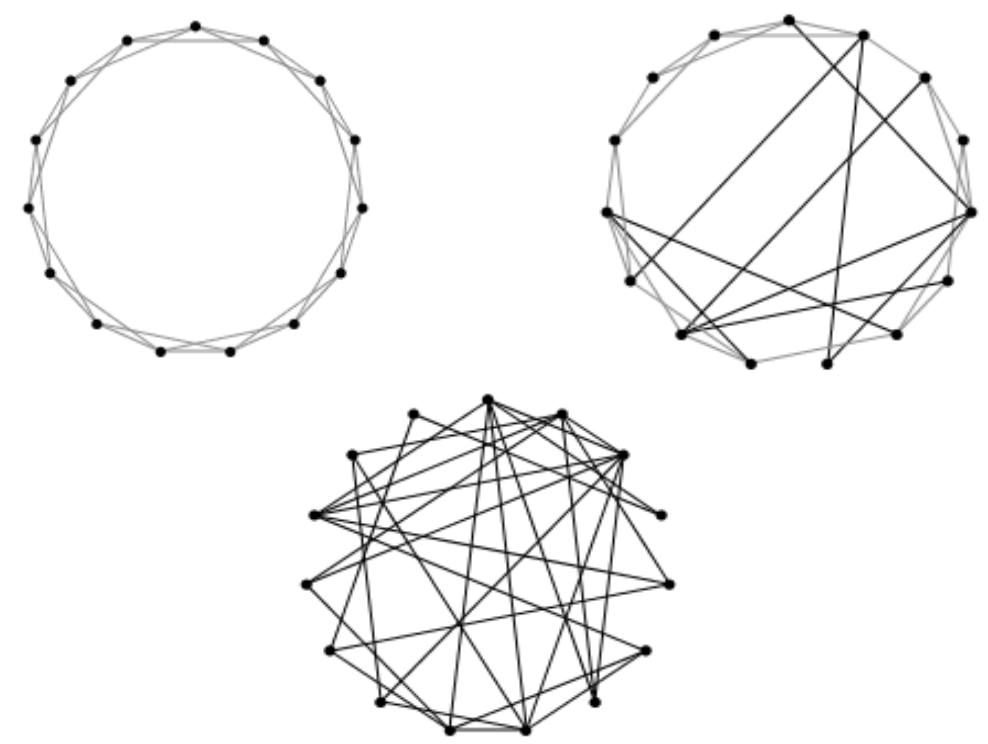
(a) Rules of the network $(\mathrm{p}=0)$
(b) WS small-world network $(0<\mathrm{p}<1)$
(c) random network $(\mathrm{p}=1)$

\section{Figure 3. Random Reconnection Process Construction of WS Small-world Network Model}

By adjusting the value of $\mathrm{p}$ ( $p \in[0,1]$ ) can be controlled from the regular network to the network completely random transition. In the small-world network model simulation results, the average path $\mathrm{L}(\mathrm{p})$ and clustering coefficient of $\mathrm{C}(\mathrm{p})$ versus $\mathrm{P}$ curve of small world network as shown in Figure 4, among them, apl and cc were said to be average in different probabilities of $\mathrm{P}$ small world network shortest path and clustering coefficient is normalized curve obtained after the treatment $(\mathrm{L}(\mathrm{p}) / \mathrm{L}(0), \mathrm{C}(\mathrm{p}) / \mathrm{C}(0)), \mathrm{N}=200, \mathrm{~K}=4$.

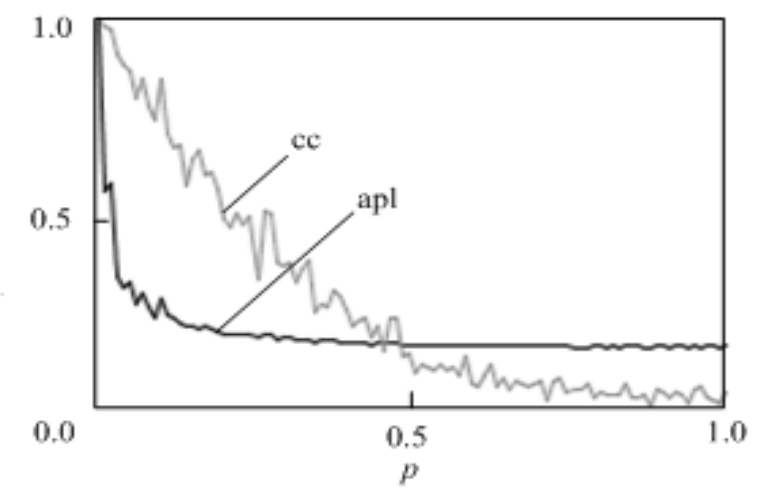

Figure 4. The Normalized Curve of $L(p)$ and $C(p)$ on WS Small-world Networks

As can be seen, the small-world networks as $\mathrm{p}$ increases, the average shortest path to a sharp decline, while the clustering coefficient decreased more slowly. Thus, small-world networks have both small-world characteristics and high clustering properties. 


\section{Construction and Analysis of Knowledge Transfer Network Model}

In the knowledge network, the interaction between the main body of knowledge through a variety of scenarios to interact, communicate and cooperate, such as project collaboration, knowledge alliances, these relationships are dependent on the network connection mode structure and knowledge transfer process. In the knowledge transfer process, the ability to release the body of knowledge ensure the knowledge receiver smoothly, the correct understanding and implementation of knowledge transfer; absorption ability can ensure the shift increased amount of knowledge, improve the efficiency of knowledge transfer. The location of the body of knowledge in the network reflects the inherent laws of knowledge transfer, knowledge exists not only within a single body, and will exist in the form of network between many different subjects, thus forming a distributed knowledge network. In such a distributed network inter-subjective knowledge diffusion and transfer, thereby enhancing the level of knowledge of the entire network and knowledge transfer capacity.

\subsection{Model Construction}

With the help of the small world network model of the reconnection algorithm to construct knowledge transfer network model. With $\mathrm{G}(\mathrm{V}, \mathrm{E})$ represents the topology of their network, $\mathrm{V}$ denotes the set of nodes $v_{i}, V=\left\{v_{1}, v_{2}, \ldots, v_{i}\right\}, \mathrm{E}$ denotes the set consisting of the relationship among nodes or links, $E=\left\{e_{1}, e_{2}, \ldots, e_{k}\right\}, e_{i j}$ represents the connection of $v_{i}$ and $v_{j}$ (namely the edge). Major network parameters include the number of nodes $\mathrm{N}$, the number of connections of nodes(namely the number of edges, $\mathrm{M}$ ), each edge corresponds to a pair of nodes. From the beginning of the cyclic regular network, set the $\mathrm{N}$ nodes, each node to the adjacent $\mathrm{K}$ node connected by total $\mathrm{K}$ edges, satisfy $N>>K>>\ln (N)>1$.For each edge, with probability p to re-connect, which would produce pNK new edges connect nodes, so that the average path is greatly decreased, while still maintaining its cluster coefficient in the level of the original.

Node attribute description: In the model, the network node attributes include: Knowledge level $\mathrm{V}$, indicates that the level of knowledge of the node $\mathrm{v}$ at time $\mathrm{t}$; knowledge transfer coefficient $\beta \in(0,1)$, which means that nodes interact during the release and transfer of knowledge ability, $\beta=0$ means that do not have the knowledge metastasis, $\beta=1$ indicates a full knowledge transfer capability; Learn absorption coefficients $\alpha \in(0,1)$, represents the node for knowledge learning and ability, $\alpha=0$ indicates that the node does not have the knowledge to learn and the ability to accept, $\alpha=$ 1 indicates that the node has full knowledge and learning and absorptive capacity; Weighted marks on each side $w_{i j}$, represents knowledge timeliness, with the simulation time increment, $w_{i j}$ gradually decreasing, when $w_{i j} \leq 0$, it means that knowledge is outdated and no longer be transferred; trust degree $x \in(0,1)$, said trust degree of a node $v_{i}$ to node $v_{j}$, it was decided at the moment when a simulation node $v_{i}$ whether the transfer of knowledge to node $v_{j}$.

Node behavior rules and algorithms described as follows: each node can affect the level of knowledge and its adjacent node directly connected members' knowledge level. Similarly, the knowledge level of each node are influenced by adjacent nodes directly connected to it, the node $v_{i}$ at time $\mathrm{t}+1$ level of knowledge depends on node $v_{j}$ knowledge potential difference. Knowledge transfer process is knowledge consists of nodes with high knowledge level to the adjacent lower level of knowledge transfer. If the level of knowledge at time $\mathrm{t} v_{i}(t)>v_{j}(t)$ and satisfy the node $v_{i}$ trust more than node $v_{j}$ 
trust, $\chi_{i}>\chi_{j}$, and knowledge is not out of date, $w_{i j}>0$,knowledge transfer takes place, or do not transfer.

Edge weights $w_{i j}$ descending algorithm is described as: edge weights randomly generated $w_{i j} \in(0,1)$, in the total simulation time, not through a simulation step, the edge weight reduction in $1 / \mathrm{t}$, at time $\mathrm{t}+1$ edge weights becomes $w_{i j}(t+1)=w_{i j}(t)-1 / T$, when $w_{i j} \leq 0$, which means that knowledge is outdated and no longer knowledge transfer, set $w_{i j}=0$.

Establish the level of knowledge $v_{t}(t)$ function of node $v_{i}$ :The moment of $\mathrm{t}+1$ node knowledge level:

$$
V_{t}(t+1)=V_{t}(t)+\sum_{j} \alpha\left[\beta V_{j}(t)-V_{i}(t)\right] \cdot
$$

The average knowledge level of knowledge transfer network: ${ }_{V}^{-}(t)=\frac{1}{N} \sum_{t} V_{i}(t)$.

Knowledge Transfer Network level of knowledge variance:

$$
\sigma(t)=\frac{1}{N}\left[\sum_{i} V_{i}^{2}(t)-N \bar{V}^{2}(t)\right] .
$$

\subsection{Simulation and Analysis of Knowledge Transfer Network Model}

4.2.1. Simulation Analysis of Small World Characteristics of Knowledge Transfer Network: In the model simulation, according to the proportion of e randomly assigned specific roles in the network, they have a high level of knowledge $\left(V_{t}(t)=1\right.$ ), complete knowledge transfer capabilities $(\beta=1)$, and they are completely trusted by other nodes $(\chi=1$ ), namely knowledge of experts; Other nodes set randomly the level of knowledge $\left(V_{t}(t)\right.$ ), knowledge transfer coefficient $(\beta)$, absorption coefficient $(\alpha)$ and trust factor coefficient $(\chi$ ) between 0 and 1 .This model set the $e=0.02$ runtime, rewiring probability $p$ step size $p=0.01$, $\mathrm{N}=200, \mathrm{~K}=4$, randomly selected $4\left(\mathrm{~N}^{*} \mathrm{e}\right)$ knowledge experts in the network model , labeled $V_{e}=1$, in each rewiring probability $\mathrm{p}$, the level of average network knowledge can take ten times the value recorded as a result of model. The normalization curve, the average shortest path and clustering coefficient with the rewiring probability $\mathrm{P}$ changes in the same rewiring probability $\mathrm{p}$ repeated ten times, Running the simulation network model, is shown in Figure 5. apl curve represents the $\mathrm{L}(\mathrm{p}) / \mathrm{L}(0)$ value, cc curve represents the value of $\mathrm{C}(\mathrm{p}) / \mathrm{C}(0)$.

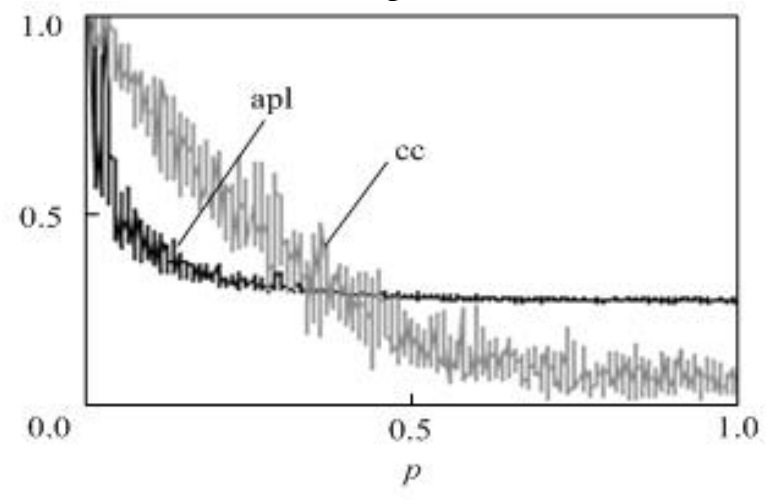

Figure 5. The Average Shortest Path and Clustering Coefficient Curve of Knowledge Transfer Network 
As can be seen, the network model conforms to the small-world network model network characteristics, as $\mathrm{p}$ increases, the sharp decline in the average shortest path, clustering coefficient decreased gentle, can be found that the amplitude of apl and cc showed a decreasing trend in the same p-value, the premise is with the increase of P. At P 0.381 , APL 0.169 small world network gradually degenerated into a random network.

The average level of knowledge based on Knowledge Transfer Network of small world network changes by the rewiring probability p of the curve shown in Figure 6, where, akl curve represents the average level of knowledge network with rewiring probability $p$ trends; var curve represents the level of network knowledge standard deviation with the rewiring probability $\mathrm{p}$ of changing trends.

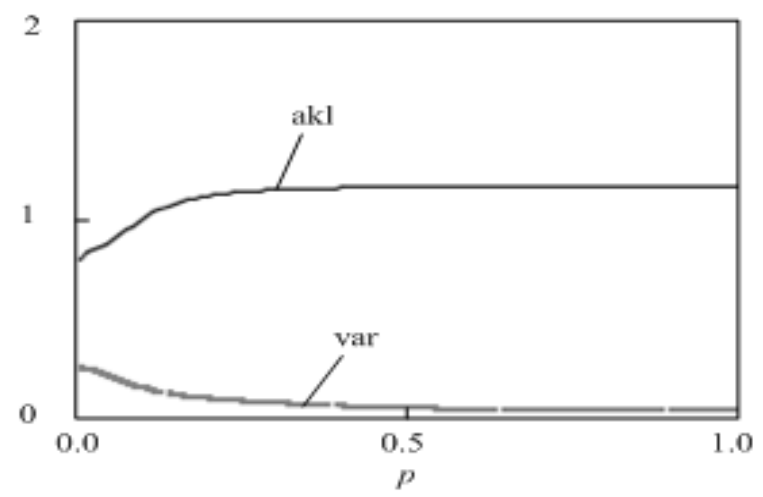

\section{Figure 6. The Evolution Curves of the Average Knowledge with Rewiring Probability $p$ for Knowledge Transfer Network}

We can obtain from the results of the simulation run, when $p \in[0.01,0.4]$, akl curve begin rising rapidly on the point $\mathrm{p}=0.01$ (Clustering coefficient $C_{(p=0.01)}$ is approximately 0.493 ,Average shortest path $L_{(p=0.01)}$ is approximately 16.079). when $p \in[0.4,1]$, the average level of network knowledge akl curve basically stable at 1.31 level,then the network average shortest path and clustering coefficient

were: $L(p \rightarrow 1) \approx \frac{\ln N}{\ln K}=3.82193, C(p \rightarrow 1) \approx \frac{N}{K}=0.02$.

This shows that small-world network characteristics have been weakened, then gradually reduced to the network structure. It can be found that from the analysis of the network knowledge level standard deviation curve Var in the $p \in[0.01,0.2]$ range, the value of $\sigma(t)$ has been dropped close to zero, indicating that the network has the high efficiency of knowledge transfer based on small-world networks of knowledge transfer.

4.2.2. Small-world Knowledge Transfer Network Capacity Simulation: Assuming in the node attributes of knowledge transfer network, knowledge transfer coefficient $\beta$, study of absorption coefficient $\alpha$, confidence coefficient $\chi$, change with the number of nodes of knowledge transfer and knowledge level in the process of the simulation period. To simplify the problem, assume that $\beta$ values and knowledge transfer to the node increases linearly, setting the evolution function of $\beta$ :

$$
\beta_{i}(t+1)=\beta_{i}(t)+\sum_{t} n_{i} / T_{\max } .
$$

Among them, $T_{\max }$ is the total simulation time, $\mathrm{n}$ is the times of knowledge transfer in the simulation time t. setting the evolution function of $\alpha$ : 


$$
\alpha_{i}(t+1)=\alpha_{i}(t)+V_{i}(t) / \sum_{i} V_{i}(t)
$$

$V_{i}(t)$ is the knowledge level at time t of node $V_{i}$.

In the simulation run time, random set the initial value of $\alpha, \beta, \chi$ in the interval $(0,1)$, the other value of simulation variables and algorithms remain unchanged. Set the maximum simulation cycle is 100,namely $T_{\max }=100$, the simulation were set rewiring probability $\mathrm{p}=0.1$ and $\mathrm{p}=0.8$,study the average level of knowledge of network nodes in this case, the average level of knowledge of the network evolution curve shown in Figure 7.

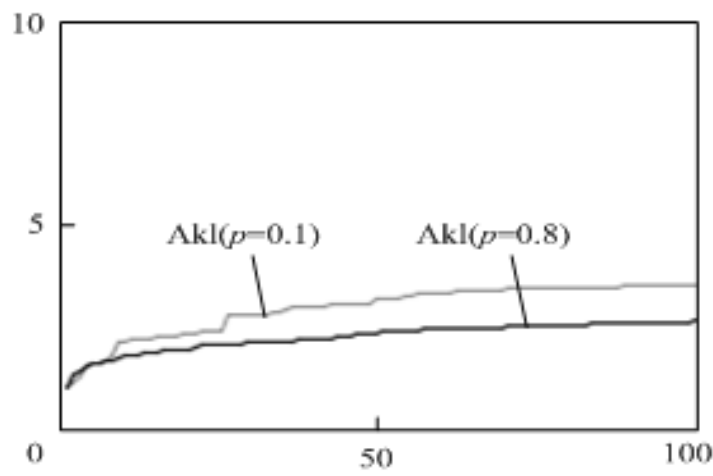

Figure 7. The Evolution Curve of Average Levels of Knowledge for Knowledge Transfer Network

As can be seen, the small world of knowledge transfer networks in knowledge transfer simulation of network nodes, the improvement of the network body's release of knowledge transfer and learning absorbing ability can increase the average level of knowledge of the entire network, but also improve the body of knowledge network transfer efficiency. And, in the knowledge transfer network model, when small-world network characteristics effect is obvious, the average level of knowledge network is greater than not obvious characteristics effect of small-world network level, indicating that small-world networking features help to improve knowledge transfer capacity network node, and impact on the behavior of the subject.

\section{Conclusion}

In this paper, based on the small world network model of knowledge transfer behavior simulation, results show that, in the small world network, subject behavior ability and organizational knowledge transfer efficiency can reach a higher level. In order to improve the efficiency of knowledge diffusion and knowledge transfer, we can improve the Inter Organizational Trust and knowledge network operation efficiency by clustering coefficient adjustment. The next step will be a detailed analysis of knowledge transfer through the main corporate network, designed to meet the characteristics of enterprise technology alliance network knowledge transfer operation and incentives.

\section{Acknowledgements}

The paper is supported by both Nature Science Foundation of jiangxi Province (no: 20132BAB201051) and Science and technology research project of jiangxi province Ministry of education (no: 11726). 


\section{References}

[1] P R. Monge, "Contractor N S. Theories of Communication Networks", New York, USA: Oxford University Press, (2003).

[2] Wangxiaofan, Lixiang and Chenguanlong, "Complex networks theory and its application", Beijing: Tsinghua University Press, (2006)

[3] R. Albert and A. L. Barabasi, "Statistical Mechanics of Complex Network. Reviews of Modern Physics, vol. 74, no. 1, (2002), pp. 47-97.

[4] F. Liljeros, C R. Edling and L A N. Amaral, "The Web of Human Sexual Contacts", Nature, vol. 411, (2001), pp. 907-908.

[5] H. Jeong, B. Tombor and R. Albert, "The Large-scale Organization of Metabolic Networks", Nature, vol. 407, (2001), pp. 651-654.

[6] D J. Watts and S H. Strogatz, "Collective Dynamics of "Small-World" Networks", Nature, vol. 393, (1998), pp. 440-442.

[7] C. Freeman, "Networks of Innovators: A Synthesis of Research Issues", Research Policy, vol. 20, no. 5, (1991), pp. 499-514.

[8] M. Granovetter, "Economic Action and Social Structure: The Pro- blem of Embeddedness", American Journal of Sociology, vol. 91, no. 3, (1985), pp. 481-510.

[9] B. Uzzi, "The Sources and Consequences of Embeddedness for the Economic Performance of Organizations: The Network Effect", American Sociological Review, vol. 61, no. 4, (1996), pp. 674-698.

[10] B. Uzzi, "Social Structure and Competition in Interfirm Networks: The Paradox of Embeddedness", Administrative Science Quarterly, vol. 42, no. 1, (1997), pp. 35-67.

[11] B. Uzzi, "Embeddedness in the Making of Financial Capital: How Social Relations and Networks Benefit Firms Seeking Financing. American Sociological Review, vol. 64, no. 4, (1999), pp. 481-505.

[12] Guolei and Xuxiaoming, "Complex networks", Shanghai: Shanghai science and technology education Press, (2006).

[13] D J. Watts, "Small Worlds: The Dynamics of Networks between Order and Randomness", Princeton, USA: Princeton University Press (1999).

[14] S. Milgram, “The Small World Problem. Psychology Today”, vol. 2, no. 1, (1967), pp. 60-67.

[15] Maqinguo, Xuqing and Liaozhengpeng, "Analysis of the influence factors on knowledge transfer", Journal of Beijing Institute of Technology (Social Sciences Edition), vol. 8, no. 1, (2006), pp. 40-43.

[16] S. Xu and H. Zou, "Analysis of Dynamics of Knowledge Transfer Based on Supernetwork", Journal of Intelligence, vol. 30, no. 7, (2011), pp. 94-98.

[17] S. Xu and B. Yang, "The Analysis of Knowledge Transfer Network Characteristic Based on Small-world Network Model", Journal of The China Society for Scientific and Technical Information, vol. 5, (2010), pp. 915-919.

[18] B. Yang, "The Knowledge Transfer Evolutionary Model and Simulation Based on the Modeling of System Dynamics", Library and Information Service, vol. 54, no. 18, (2010), pp. 89-95.

[19] Netlogo[EB/OL]..http://ccl.north-western.edu/netlogo(2011-04-20)

[20] Zagreb and Croatia, "A introduction to agent based modeling and simulation of social processes [EB/OL]”, http://arxiv.org/ftp/cond-mat/papers/0409/0409312.pdf. (2011-04-20) 
International Journal of Multimedia and Ubiquitous Engineering

Vol.10, No.5 (2015) 